\title{
Differential Regulation of Anthocyanin Synthesis in Apple Peel under Different Sunlight Intensities
}

\author{
Weifeng Chen, Mengxia Zhang, Guojing Zhang, Pengmin Li * and Fengwang Ma
}

State Key Laboratory of Crop Stress Biology for Arid Areas/Shaanxi Key Laboratory of Apple, College of Horticulture, Northwest A\&F University, Yangling 712100, China; c4611475@nwsuaf.edu.cn (W.C.); mz458@cornell.edu (M.Z.); zhanggj1230@163.com (G.Z.); fwm64@nwsuaf.edu.cn (F.M.)

* Correspondence: Lipm@nwsuaf.edu.cn

Received: 9 November 2019; Accepted: 29 November 2019; Published: 1 December 2019

\begin{abstract}
Sunlight radiation is a main environmental factor which affects anthocyanin synthesis. To clarify the regulatory mechanism of sunlight on the synthesis of anthocyanin in apple peel, bagged apples were exposed to diverse intensities of sunlight through different shading treatments. Under an increased solar ultraviolet-B (UV-B) light intensity, the concentration of anthocyanin in apple peels was consistent with the Michaelis-Menten equation. Under lower sunlight intensities, diphenyleneiodonium chloride (DPI, an inhibitor of plasma membrane $\mathrm{NAD}(\mathrm{P}) \mathrm{H}$ oxidase) treatment increased both the concentration of cyanidin-3-glycoside and the activity of dihydroflavonol 4-reductase (DFR). However, under higher sunlight intensities, DPI treatment decreased the concentrations of cyanidin-3-glycoside and quercetin-3-glycoside, as well as the activities of DFR and UDP-glycose: flavonoid 3-O-glycosyltransferase (UFGT). These results indicate that, under low sunlight intensity, anthocyanin synthesis in apple peel was limited by the supply of the substrate cyanidin, which was regulated by the DFR activity. Nevertheless, after exposure to high sunlight intensity, the anthocyanin produced in the apple peel was dependent on UFGT activity.
\end{abstract}

Keywords: anthocyanin synthesis; sunlight; UV-B; DFR; UFGT; apple

\section{Introduction}

Apples are an important agricultural crop and anthocyanins are the visible sign of apple maturity [1]. Anthocyanins belong to the reddish secondary metabolites, which consist of anthocyanidin and glycoside. They can increase the commerciality of various health foods and extend their shelf life $[2,3]$. Anthocyanins act as visual signals to attract insects for pollination and agents for seed dispersal [4], and also function as anti-oxidants, scavenging free radicals and protecting plant tissues against both biotic and abiotic stresses [5]. These compounds also have a positive effect on human health, serving as vasodilators and reducing the risk of myocardial infarction $[6,7]$. Anthocyanins have potential anti-diabetic properties; in addition, anthocyanin-rich foods can reduce starch consumption and delay glucose absorption by inhibiting alpha-amylase and alpha-glucosidase [8]. Anthocyanins can also benefit our bodies in terms of neuroprotection, vision improvement, anti-inflammatory effects, antimicrobial activity, chemoprevention, and cancer protection [9-11].

The synthesis of anthocyanins is involved in the phenylpropanoid metabolic pathway. Recently, the regulatory mechanisms of anthocyanin synthesis were reported [12-18]. Sunlight is one of the environmental factors regulating gene expression and plant development, and ultraviolet-B (UV-B) is considered to be a major factor to increase the synthesis of anthocyanins and flavonoids in plants [12,15]. UV-B induced photomorphogenesis is initiated by the specific photoreceptor, UV RESISTANCE LOCUS 8 (UVR8), which utilizes its tryptophan residue as an internal chromophore to sense UV-B [18]. UVR8 regulates the light signaling factors E3 ubiquitin ligase CONSTITUTIVELY PHOTOMORPHOGENIC1 
(COP1) and bZIP transcription factor ELONGATED HYPOGOTYL5 (HY5) [18,19]. When COP1 and HY5 are combined with the MYB transcription factor, they regulate anthocyanin accumulation [20-22].

The biosynthesis of anthocyanins in apple skin mainly depends on the MYB-bHLH-WD40 (MBW) complex [23,24]. At low temperature and under artificial UV-B conditions, MdbHLH3 binds to promoters of the anthocyanin synthesis genes MdDFR and MdUFGT to activate their expressions in apple fruit [25]. MYB10 regulates the synthesis of anthocyanins by regulating the activity of UDP-glycose: flavonoid 3-O-glycosyltransferase (UFGT) under partly-filtered solar UV irradiation conditions [12]. Previous apple studies have shown that chalcone synthase (CHS) is not the rate-limiting step in anthocyanin synthesis [26,27], but that its overexpression can significantly increase the accumulation of anthocyanins and upregulate the expression levels of AtDFR and AtANS under strong light in Arabidopsis [28]. Our previous studies demonstrated that chalcone isomerase (CHI) and flavanone 3-hydroxylase (F3H) were not key enzymes controlling the synthesis of anthocyanins and flavonols in apple [29]. Hydroflavonol 4-reductase (DFR) is a key enzyme in the biosynthesis of anthocyanins and has been extensively studied in many plants [30-32]. In apple peels, CHS, F3H, DFR, ANS, and UFGT achieved higher expression levels in UV-B and low-temperature conditions [33]. Previous studies have provided direct evidence that MYBA activates the DFR promoter in blueberry plants and the ANS promoter in apple peel $[34,35]$. The UV-B-specific perception and signaling pathways involving UVR8/COP1/HY5 have been suggested to work under narrow-band, low UV-B conditions, while the activation of reactive oxygen species (ROS)-mediated signaling, DNA damage response phytohormones, and mitogen-activated protein kinase (MAPK) signaling are promoted under broad-band, high UV-B conditions [36,37]. Through UV-light attenuation and diphenyleneiodonium chloride (DPI, an inhibitor of plasma membrane NADPH oxidase) treatment, it was found that the anthocyanin synthesis in 'Golden Delicious' was regulated by the ROS produced via plasma membrane NADPH oxidase [12]. Therefore, different UV-B irradiation conditions might regulate anthocyanin synthesis through different signaling pathways and structural genes.

In natural conditions, the radiation intensity of sunlight changes throughout the day. Due to shading of the tree canopy, each fruit receives a diverse intensity of UV-B radiation. With this variability, the structural genes which play important roles in the regulation of anthocyanin synthesis might also be different. In this study, we analyzed anthocyanin biosynthesis in both 'Fuji' and 'Red Delicious' apples, treated with diphenyleneiodonium chloride (DPI) which is an $\mathrm{NAD}(\mathrm{P}) \mathrm{H}$ oxidase inhibitor that can restrained ROS to affect anthocyanin synthesis, upon exposure to diverse sunlight intensities, in order to gain further insights into the regulation of anthocyanin synthesis.

\section{Results}

\subsection{Anthocyanin Concentrations under Different Sunlight Intensities Fit with Michaelis-Menten Equation}

Through different shading treatments, the apple fruits were exposed to different intensities of sunlight. With increased solar UV-B light intensity, the concentration of total anthocyanins (cyanidin-3-galacotoside plus cyanidin-3-glucoside) in both 'Fuji' and 'Red Delicious' apple peels initially increased in a linear pattern, and then gradually leveled off in either 2016 or 2017 (Figure 1A-C). Moreover, the concentrations of anthocyanins under different light intensities showed a good fit with the Michaelis-Menten equation, at $\mathrm{R}^{2}$ values (the fitting formula) of $0.988,0.979$, and 0.992 . 


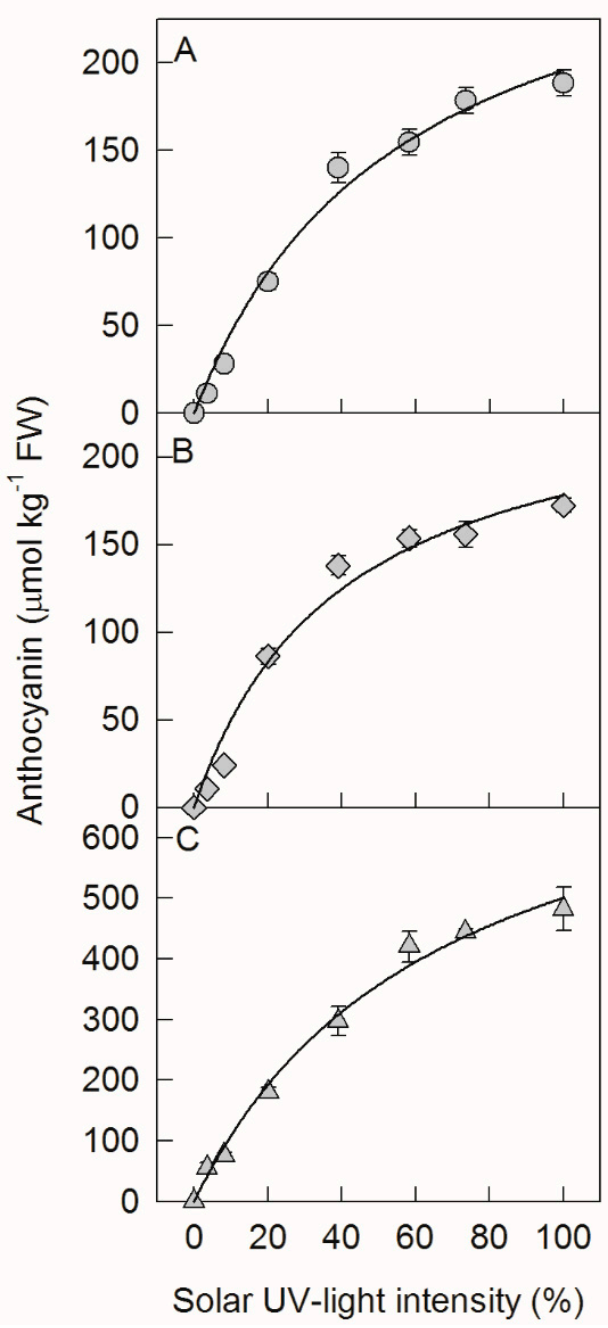

Figure 1. Concentrations of anthocyanin in 'Fuji' apple peel in 2016 (A) and 2017 (B) and 'Red Delicious' apple peel in 2017 (C) after exposing bagged fruits to diverse sunlight intensities. Each data point represents mean $\pm \mathrm{SE}(n=5)$. The fitting formula were $\mathrm{y}=\frac{305.4 \mathrm{x}}{55.9+\mathrm{x}}, \mathrm{R}^{2}=0.988(\mathrm{~A}), \mathrm{y}=\frac{249.6 \mathrm{x}}{40.1+\mathrm{x}}$, $\mathrm{R}^{2}=0.979(\mathbf{B})$, and $\mathrm{y}=\frac{836.9 \mathrm{x}}{67.1+\mathrm{x}}, \mathrm{R}^{2}=0.992(\mathbf{C})$, respectively.

\subsection{Analysis of Anthocyanin and Flavonol Concentrations under Different Sunlight Intensities}

The concentrations of flavonol, quercetin-3-galactoside, and quercetin-3-glucoside changed in similar patterns to cyanidin-3-glycoside in 'Fuji' apple peel as the solar UV-B light intensity increased (Figure 2C,D). When the light intensity was below $50 \%$, DPI treatment increased the concentration of cyanidin-3-glycoside, but did not change the concentration of quercetin-3-glycoside in 'Fuji' apple peel. When the solar light intensity was over $50 \%$, DPI treatment decreased the concentrations of both cyanidin-3-glycoside and quercetin-3-glycoside. 


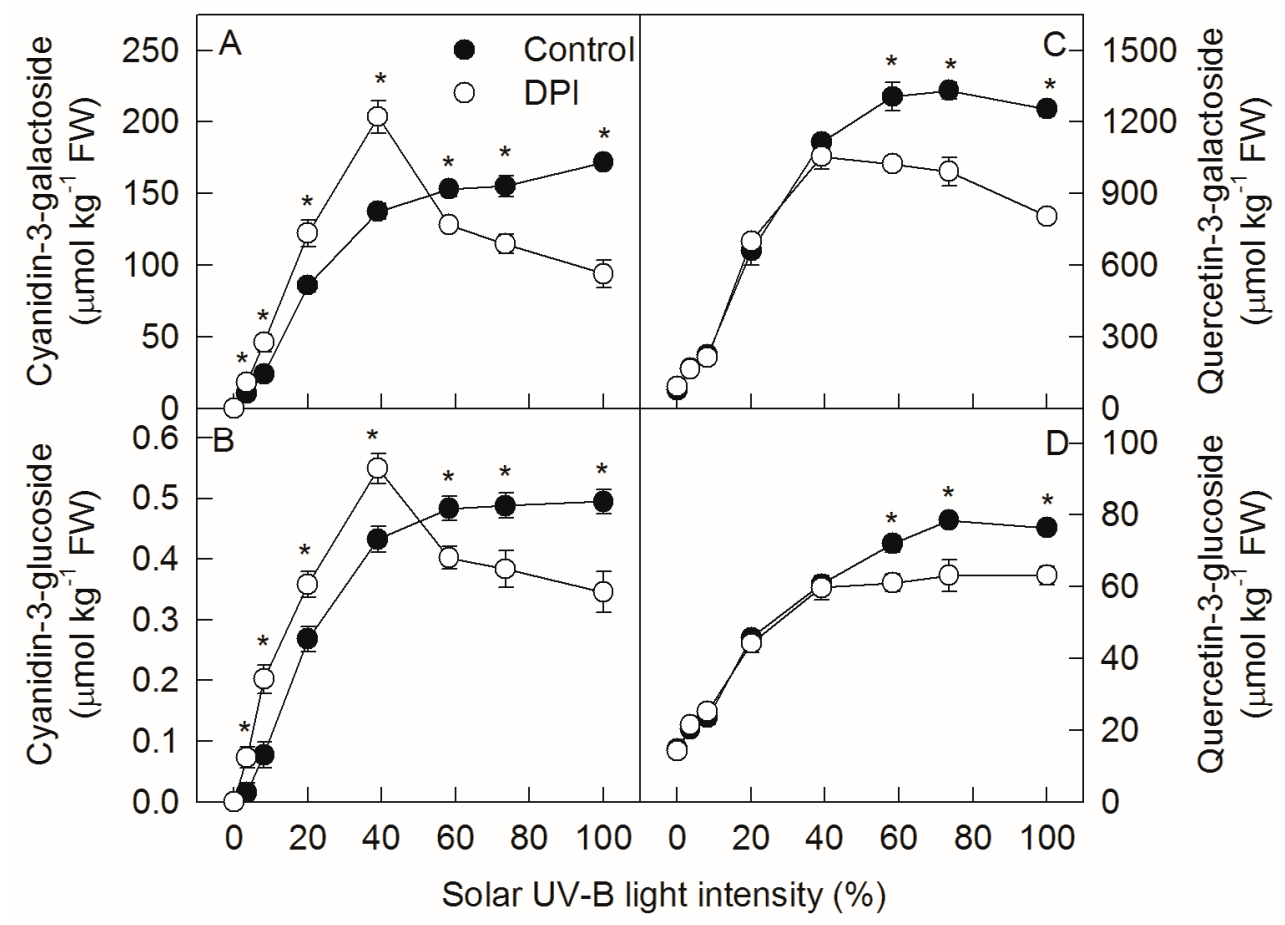

Figure 2. Concentrations of cyanidin-3-galactoside (A), cyanidin-3-glucoside (B), quercetin3-galactoside (C), and quercetin-3-glucoside (D) in 'Fuji' apple peels after exposing bagged fruits, with or without diphenyleneiodonium chloride (DPI) treatment, to diverse sunlight intensities. Each data point represents mean $\pm \mathrm{SE}(n=5)$. The asterisk indicates a significant difference between DPI treatment and no DPI treatment at $p<0.05$ (t-test).

\subsection{Analysis of Nucleotide Sugar Concentrations under Different Sunlight Intensities}

As nucleotide sugar provides glycosyl group for anthocyanin synthesis, the concentration of UDP-galactose was also assayed. The concentration of UDP-galactose in 'Fuji' apple peel declined and then remained at a stable level along with the increasing of solar UV-B light intensity (Figure 3). Treatment with DPI did not change the concentration of UDP-galactose.

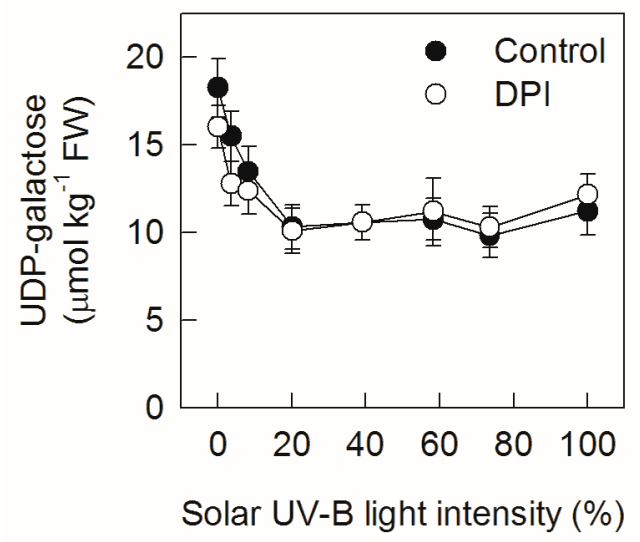

Figure 3. Concentrations of UDP-galactose in 'Fuji' apple peels after exposing bagged fruits, with or without DPI treatment, to diverse sunlight intensities. Each data point represents mean $\pm \operatorname{SE}(n=5)$.

\subsection{Analyze Gene Expression Levels under Different Sunlight Intensities}

Transcription levels of MdUVR8, MdHY5, MdCOP1, MdMYB10, MdCHS, MdCHI, MdDFR, MdANS, and MdUFGT in 'Fuji' apple peel initially increased and then remained unchanged with increasing 
solar UV-B light intensity, except for MdDFR and MdUFGT, which slightly decreased under higher light intensities (Figure 4A-H and Figure S1). DPI treatment did not change the gene levels, regardless of sunlight intensity, except for MdHY5 and MdCOP1, which increased upon full exposure.

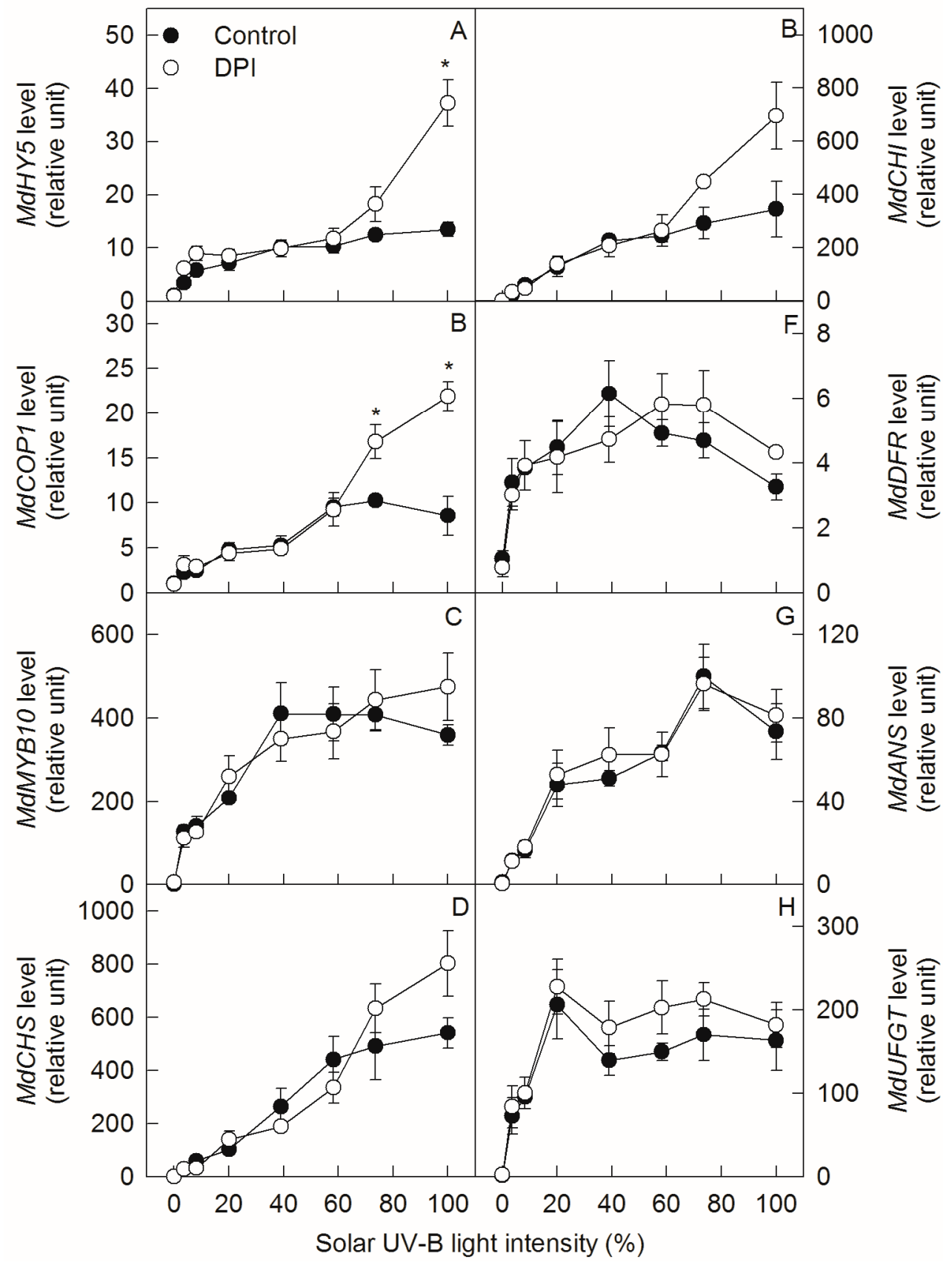

Figure 4. Transcription levels of MdHY5 (A), MdCOP1 (B), MdMYB10 (C), MdCHS (D), MdCHI (E), $\operatorname{MdDFR}(\mathbf{F}), \operatorname{MdANS}(\mathbf{G})$, and MdUFGT (H) in 'Fuji' apple peels after exposing bagged fruits, with or without DPI treatment, to diverse sunlight intensities. Each data point represents mean $\pm \operatorname{SE}(n=5)$. The asterisk indicates a significant difference between DPI treatment and no DPI treatment at $p<0.05$ ( $t$-test).

\subsection{Analysis of Enzyme Activities under Different Sunlight Intensities}

The activities of CHS, DFR, and ANS in 'Fuji' apple peel continuously increased in accordance with increasing solar UV-B light intensity, but the CHI activity remained unchanged (Figure 5A-D). The UFGT activity increased linearly and remained steady with increasing intensity, but then slightly 
declined upon full exposure (Figure 5E). When the solar UV-B light intensity was below 50\%, DPI treatment significantly increased DFR activity. When the solar UV-B light intensity was over $50 \%$, DPI treatment decreased the activities of both DFR and UFGT. DPI treatment did not affect the activities of CHS, CHI, or ANS in 'Fuji' apple peel.

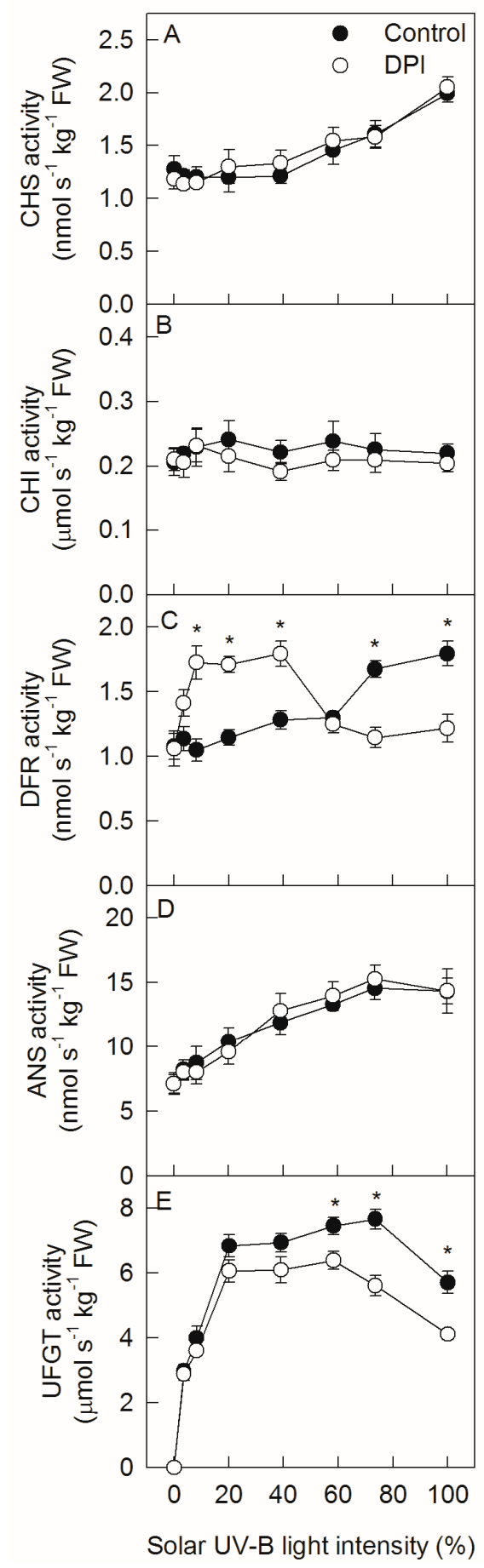

Figure 5. Activities of chalcone synthase (CHS, (A)), chalcone isomerase (CHI, (B)), dihydroflavonol 4-reductase (DFR, (C)), anthocyanidin synthase (ANS, (D)), and UDP-glycose: flavonoid 3-O-glycosyltransferase (UFGT, (E)) in 'Fuji' apple peels after exposing bagged fruits, with or without DPI treatment, to diverse sunlight intensities. Each data point represents mean $\pm \mathrm{SE}(n=5)$. The asterisk indicates a significant difference between DPI treatment and no DPI treatment at $p<0.05$ ( $t$-test). 


\section{Discussion}

In apple peel, the predominant anthocyanin component is cyanidin-3-galactoside [29,38,39]. The synthesis of cyanidin-3-galactoside was catalyzed by UFGT, namely, cyanidin + UDP-galactose $\stackrel{\text { UFGT }}{\rightarrow}$ cyanidin-3-galacoside + UDP. The produced cyanidin-3-galacoside (A) is $\mathrm{A}=V \times \mathrm{t}$, where $V$ is the rate of cyanidin-3-galactoside synthesis, and $\mathrm{t}$ is time. According to the Michaelis-Menten equation, $V=\frac{V \max [S]}{K m+[S]}$, and $\mathrm{A}=\frac{V \max [S]}{K m+[S]} \times \mathrm{t}$. In this study, apples were exposed to different sunlight intensities for the same period of time. Thus, A at different light intensities was determined by $V$. Under low light intensity, $V \mathrm{O}=\frac{V \max [S]}{K m}$, and $\mathrm{A}=\frac{V \max [S]}{K m} \times \mathrm{t}$. The produced cyanidin-3-galactoside depends on the concentration of the substrate [S]. Under high light intensity, $V_{\mathrm{o}}=V \max$, namely, $\mathrm{A}=V \max$ $\times t$, and the produced cyanidin-3-galactoside depends on UFGT activity. Clearly, according to the Michaelis-Menten equation, the regulatory mechanism of anthocyanin synthesis in apple peel was different upon the exposure of bagged apples to diverse intensities of solar UV-B. When the solar UV-B light intensity was relatively low (below 50\%), anthocyanin synthesis was limited by the supply of the substrate, whereas it was limited by UFGT activity when the solar UV-B light intensity was relatively high (over 50\%, Figures 1 and 5).

In our previous studies, after exposing bagged 'Golden Delicious' apples to sunlight, DPI (an inhibitor of plasma membrane oxidase) treatment inhibited the production of reactive oxygen species (ROS) via plasma membrane NADPH oxidase and the signaling pathway, reducing the expressions of MdMYB10 and MdUFGT, the enzyme activity of UFGT, and the synthesis of anthocyanin in apple peel [12]. In this study, DPI treatment also inhibited anthocyanin synthesis in 'Fuji' apple peel when the bagged fruits were exposed to relatively high sunlight intensities (Figure 2). However, DPI did not affect the expression levels of MdMYB10 and MdUFGT, but inhibited the enzyme activities of DFR and UFGT (Figures 4 and 5). The different gene expression and enzyme activity responses might be related to the different cultivar properties, as 'Golden Delicious' is a non-red cultivar and 'Fuji' is a red cultivar. UFGT catalyzes the glycosylation of both cyanidin and quercetin in apple peel $[27,40]$, while DFR is only involved in the synthesis of cyanidin [30-32]. Therefore, under relatively high sunlight conditions, the lower concentrations of quercetin-3-glycoside in DPI-treated 'Fuji' peels suggest that DPI may inhibit the synthesis of anthocyanin mainly by affecting the activity of UFGT (Figure 2). This is also consistent with the conclusion derived from the Michaelis-Menten equation, as mentioned above.

Interestingly, DPI treatment significantly increased the concentration of anthocyanin in 'Fuji' fruit peels after exposing the bagged fruits to relatively low sunlight conditions (Figure 2). This indicates that DPI treatment might have different effects on the regulation of anthocyanin synthesis in apple peel under diverse sunlight intensities. Indeed, DPI treatment did not inhibit the enzyme activity of UFGT, but increased that of DFR under relatively low sunlight conditions (Figure 5). DFR and ANS catalyze the synthesis of cyanidin, so the relatively higher DFR activity may provide more cyanidin for anthocyanin synthesis. According to the Michaelis-Menten equation, the synthesis of anthocyanin is limited by the substrate concentration under relatively low sunlight conditions (Figure 1). Since DPI treatment did not affect the concentration of UDP-Gal and the expression level and enzyme activity of ANS in apple peel (Figure 3, Figure 4G and Figure 5D), the supply of cyanidin catalyzed by DFR should be the limitation of anthocyanin synthesis under conditions of relatively low sunlight. The concentration of quercetin-3-glycoside was not affected by DPI treatment in relatively low sunlight conditions, indicating that other genes (enzymes) in the pathway shared by both anthocyanin and quercetin synthesis were not affected by DPI treatment. It is unclear why DPI treatment improved DFR activity under relatively low sunlight conditions. Wargent and Jordan (2013) suggested that anthocyanin synthesis is regulated through different signaling pathways under different UV-B irradiation conditions [37]. As anthocyanin was more sensitive to ROS than other flavonoid compounds in apple peel [41], it is perhaps that the more accumulated anthocyanins in DPI treated fruit peels were attributed to fewer ROS produced via plasma membrane NADPH oxidase. However, at first, under relatively low sunlight conditions, 
the produced ROS was also at low levels. Secondly, although ROS such as hydrogen peroxide may go through the cellular membrane, the enzymatic and non-enzymatic antioxidant systems in cytosol would be the first defense line against ROS prior to diffusion from the extracellular site of genesis to the vacuole where the anthocyanins are mainly located. In our previous studies, even at high light conditions, anthocyanin was not involved in the detoxification of ROS in pear peel [42].

In conclusion, the regulatory mechanisms of anthocyanin synthesis in apple peel were different under diverse sunlight intensities. This might be the reason that previous studies have shown different limiting factors for anthocyanin synthesis in apple peel [12,20,34]. Under relatively low sunlight intensities, anthocyanin synthesis in apple peel was limited by the supply of the substrate cyanidin, which was regulated by the activity of DFR. However, under relatively high sunlight intensities, the produced anthocyanin is dependent on UFGT activity (Figure 6). These results might be useful for the development of new biotechnological strategies for improving the quality and market value of apples.

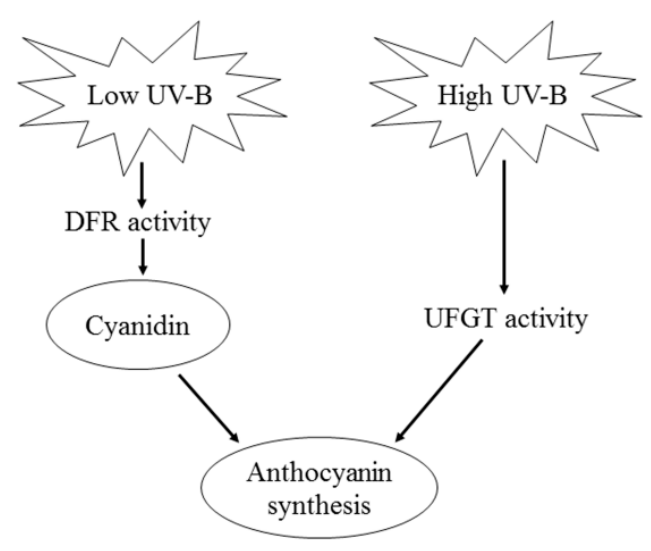

Figure 6. A regulation model of anthocyanin synthesis in apple peel under different sunlight intensities.

\section{Materials and Methods}

\subsection{Plant Materials}

Two apple cultivars (Malus domestica Borkh.), 'Fuji' and 'Red Delicious', were used in this study. 'Fuji' trees were field-grown at a spacing of $2.5 \mathrm{~m} \times 3.5 \mathrm{~m}$ in Qianxian $\left(34.53^{\circ} \mathrm{N}, 108.23^{\circ} \mathrm{E}\right.$; elevation $700 \mathrm{~m}$ ), Shaanxi, China. 'Red Delicious' trees were field-grown at a spacing of $2.5 \mathrm{~m} \times 3.5 \mathrm{~m}$ in Luochuan $\left(35.76^{\circ} \mathrm{N}, 109.42^{\circ} \mathrm{E}\right.$; elevation $\left.1033 \mathrm{~m}\right)$, Shaanxi, China. The plants were approximately $4 \mathrm{~m}$ tall with a central leader, and were grown using high quality horticultural cultivation techniques standard with disease and pest control. The apple fruits were bagged with light impermeable double-layer paper bags (the outer layer was colored yellow on the outside and black on the inside, the inner layer was red). The 'Fuji' fruits were bagged on 2 May 2016 and 3 May 2017 (approximately 30 days after full bloom), and were then harvested on 26 September 2016 and 27 September 2017 (approximately 176 days after full bloom), respectively. The 'Red Delicious' fruits were bagged on 10 May 2017 (approximately 30 days after full bloom), and were then harvested on 1 September 2017 (approximately 144 days after full bloom). Five replicates (ten fruits for each replicate) of the bagged fruits were sampled (three trees per replicate, 15 trees total) without removing the bags, to avoid exposure to light before chemical and sunlight exposure treatments.

\subsection{Chemical and Diverse Intensities Solar UV-B Light Exposure Treatments}

An external solar electric quantum meter (Spectrum Technologies Company, Chicago, Illinois, USA) and an ultraviolet radiometer (Instrument Factory of Beijing Normal University, Beijing, China) were used to measure visible and ultraviolet light transmittance, respectively. The data were collected at 07:00, 09:00, 11:00, 13:00, 15:00, 17:00, and 19:00 $\mathrm{h}$ in an open place on a sunny day. The strongest 
solar light intensity, recorded at 13:00, was $1800 \pm 50 \mu \mathrm{molm}^{-2} \mathrm{~s}^{-1}$ photon flux density and set as $100 \%$ (Figure 7). The air temperature and humidity were $28 \pm 1{ }^{\circ} \mathrm{C}$ and $45 \%$, respectively, at midday during the treatments.

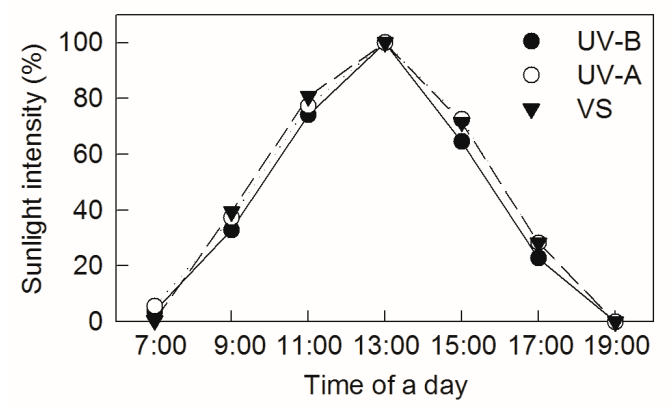

Figure 7. Sunlight intensity on a sunny day. The strongest sunlight intensity at 13:00 was set as 100\%. VS represents visible sight.

Chemical treatments were carried out in 2017 using 'Fuji' fruits. Fruits removed from their bags were sequentially immersed in a $10 \mu \mathrm{M}$ diphenyleneiodonium chloride (DPI, Sigma-Aldrich, St. Louis, $\mathrm{MO}$, USA, dissolved in $0.2 \%$ ethanol) or $0.2 \%$ ethanol solution overnight before sunlight exposure. Before sunrise, six groups of apples were placed on wet cotton gauze under diverse layers and meshes of nylon net in an open space for exposure to sunlight, with $20 \mathrm{~cm}$ of ground clearance. The relative transmission solar UV-B light intensities were 3.5\%, 8.2\%, 20.0\%, 39.0\%, 58.3\%, and 73.5\%, with shading by 16 layers of 200 mesh screen, 16 layers of 100 mesh screen, 8 layers of 60 mesh screen, 4 layers of 60 mesh screen, 2 layers of 60 mesh screen, or 1 layer of 15 mesh screen, respectively. Other two groups of apples were put into a dark box for dark treatment and on the ground for full exposure, respectively.

Apple peel samples were collected after one week of irradiation. The peels were immediately frozen in liquid nitrogen, then ground to powder and mixed in liquid nitrogen circumstance with an A11 grinder from IKA ${ }^{\circledR}$ Works (VWR, Radnor, PA, USA), and finally stored at $-80^{\circ} \mathrm{C}$ until further analysis.

\subsection{Analysis of Flavonoid Compounds}

The extraction and analysis of flavonoid compounds were carried out as described by Li et al. [29]. Briefly, the frozen tissue powder $(0.5 \mathrm{~g})$ was ground in $1.5 \mathrm{~mL}$ phenolic compound, extracting a solution containing $70 \%$ methanol and $2 \%$ formic acid at $0-4{ }^{\circ} \mathrm{C}$. After centrifugation at $10,000 \mathrm{~g}$ for $20 \mathrm{~min}$, the supernatant was passed through a $0.22-\mu \mathrm{m}$ syringe filter prior to analysis.

The Inertsil ODS-3 column ( $5.0 \mu \mathrm{m}$ particle size, $4.6 \mathrm{~mm} \times 250 \mathrm{~mm}$, GL Sciences Inc., Tokyo, Japan) was used for the separation, preceded by an Inertsil ODS-3 Guard Column. A 10- $\mu$ L sample of the filtered supernatant was injected into an LC-20A Liquid Chromatograph with a diode array detector (Shimadzu Corporation, Tokyo, Japan). Solvent A consisted of $10 \%$ formic acid (11.36\% $88 \%$ formic acid) in water and solvent $B$ consisted of $10 \%$ formic acid and $1.36 \%$ water (11.36\% 88\% formic acid) in acetonitrile. The gradients used were $95 \% \mathrm{~A}(0 \mathrm{~min}), 85 \% \mathrm{~A}(25 \mathrm{~min}), 78 \% \mathrm{~A}(42 \mathrm{~min}), 64 \% \mathrm{~A}(60 \mathrm{~min})$, and $95 \% \mathrm{~A}(65 \mathrm{~min})$ with a post-run time of $10 \mathrm{~min}$. The flow rate was $1.0 \mathrm{~mL} \mathrm{~min}^{-1}$, and the column temperature was set at $35^{\circ} \mathrm{C}$. Flavonoid compounds were detected at $365 \mathrm{~nm}$ for flavonols and $520 \mathrm{~nm}$ for anthocyanins.

\subsection{Analysis of Nucleotide Sugar}

The extraction and analysis of nucleotide sugar were carried out as described by Li et al. [29]. Briefly, frozen tissues $(0.5 \mathrm{~g})$ were extracted with $1.2 \mathrm{~mL}$ of $6 \% \mathrm{HClO}_{4}$ and $5 \%$ insoluble polyvinylpolypyrrolidone (PVPP) at $0-4{ }^{\circ} \mathrm{C}$. After centrifugation at $12,000 \times g$ for $10 \mathrm{~min}, 0.8 \mathrm{~mL}$ of the supernatant were transferred to another Eppendorf tube and then neutralized with $55 \mu \mathrm{L}$ of $5 \mathrm{M} \mathrm{K}_{2} \mathrm{CO}_{3}$. 
The resulting potassium chlorate was removed by $5 \mathrm{~min}$ centrifugation at $12,000 \times g$. The supernatant was used to measure metabolites.

The UDP-galactose was separated and quantified using a LC-20A Liquid Chromatograph. The Inertsil ODS-3 column was used in the separation, preceded by an Inertsil ODS-3 Guard Column. A $10-\mu \mathrm{L}$ sample of the filtered supernatant was injected into the liquid chromatograph. The mobile

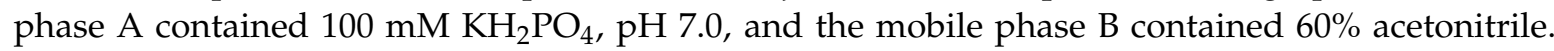
The gradient for high-performance liquid chromatograph (HPLC) analysis was performed as follows (total $43 \mathrm{~min}): 100 \%$ A (0 min), 100\% A (15 min), 90\% A (24 min), 0\% A (25 min), 0\% A (35 min), $100 \% \mathrm{~A}(36 \mathrm{~min})$, and $100 \% \mathrm{~A}$ (43 $\mathrm{min})$. The flow rate was changed as follows: $0.3 \mathrm{~mL} \mathrm{~min}^{-1}$ (0 min), $0.3 \mathrm{~mL} \mathrm{~min}^{-1}$ (13 $\left.\mathrm{min}\right), 1 \mathrm{~mL} \mathrm{\operatorname {min } ^ { - 1 }}(15 \mathrm{~min})$, and $1 \mathrm{~mL} \mathrm{~min}^{-1}$ (43 $\left.\mathrm{min}\right)$. The post run-time was $2 \mathrm{~min}$, the column temperature was at $35^{\circ} \mathrm{C}$, and the detection wavelength was $260 \mathrm{~nm}$.

\subsection{Quantitative Real-Time Polymerase Chain Reaction (qRT-PCR) Expression Analysis}

Total RNA was isolated using the SDS-phenol method according to Malnoy et al. [43]. First-strand cDNA was synthesized using the PrimeScript ${ }^{\text {TM }}$ RT reagent Kit (Takara, Dalian, China), according to the manufacturer's protocol. All qRT-PCR experiments were performed with the Bio-Rad CFX96 system (Bio-Rad Laboratories, Hercules, CA, USA) with 0.1-mL 8-tube strips, using SYBR Premix Ex TaqTM II (Takara, Dalian, China). MdActin was used as the internal reference gene. The PCR amplification program was $95^{\circ} \mathrm{C}$ for $3 \mathrm{~min}, 39$ cycles of $95^{\circ} \mathrm{C}$ for $10 \mathrm{~s}$, and $57^{\circ} \mathrm{C}$ for $30 \mathrm{~s}$, followed by a melting curve analysis program. The primers for MdActin, MdUVR8, MdHY5, MdCOP1, MdMYB10, MdCHS, MdCHI, MdDFR, MdANS, and MdUFGT are shown in Table S1.

\subsection{Analysis of Key Enzyme Activities in Anthocyanin Synthesis}

Enzymes were extracted as follows: For CHI (EC 5.5.1.6) and DFR (EC 1.1.1.219), $0.5 \mathrm{~g}$ of frozen apple peel tissue were ground in $1.8 \mathrm{~mL}$ of $100 \mathrm{mM}$ Tris- $\mathrm{HCl}$ buffer ( $\mathrm{pH}$ 7.5) containing $14 \mathrm{mM}$ $\beta$-mercaptoethanol, $5 \mathrm{mM}$ dithiothreitol (DTT), $1 \%$ bovine serum albumin (BSA), $0.5 \%$ Triton X-100, and 5\% PVPP. For CHS (EC 2.3.1.74), $1 \mathrm{~g}$ of frozen tissue was homogenized with 5\% PVPP in $2 \mathrm{~mL}$ of $100 \mathrm{mM}$ sodium phosphate buffer ( $\mathrm{pH}$ 6.8) containing $14 \mathrm{mM} \beta$-mercaptoethanol, $5 \mathrm{mM}$ DTT, $40 \mathrm{mM}$ sodium ascorbate, $3 \mathrm{mM}$ EDTA, $10 \mu \mathrm{M}$ leupeptin, and $1 \%$ BSA at $0-4{ }^{\circ} \mathrm{C}$. For anthocyanin synthase (ANS, EC 1.14.11.19) and UFGT (EC 2.4.1.91), $1 \mathrm{~g}$ of frozen tissue was homogenized with $5 \%$ PVPP in $2 \mathrm{~mL} 100 \mathrm{mM}$ Tris- $\mathrm{HCl}$ buffer ( $\mathrm{pH}$ 8.0) containing $14 \mathrm{mM} \beta$-mercaptoethanol, $5 \mathrm{mM} \mathrm{DTT}, 5 \mathrm{mM}$ EDTA, $15 \mathrm{mM} \mathrm{MgCl}_{2}, 0.5 \%$ Triton X-100, and 1\% BSA. After centrifugation, the enzyme extract was concentrated with ammonium sulfate, as described above. All of the above enzyme extracts were desalted by passage through PD10 columns and were then immediately used for enzyme assays. CHS, CHI, DFR, ANS, and UFGT were assayed as described by Li et al. [29].

\subsection{Statistical Analysis}

All data were analyzed with $t$-tests $(p<0.05)$ using SPSS 16.0 (SPSS Inc., Chicago, IL, USA).

Supplementary Materials: The following are available online at http://www.mdpi.com/1422-0067/20/23/6060/s1.

Author Contributions: P.L. designed this work. W.C. and M.Z. performed most of the experiments and W.C. wrote the draft of manuscript. G.Z. performed parts of the experiments in the laboratory. P.L. and F.M. revised the manuscript. All authors have read and approved the final manuscript.

Funding: This research received no external funding.

Conflicts of Interest: The authors declare that there is no conflict of interest. 


\section{Abbreviations}

$\begin{array}{ll}\text { CHS } & \text { chalcone synthase } \\ \text { CHI } & \text { chalcone isomerase } \\ \text { F3H } & \text { flavanone 3-hydroxylase } \\ \text { DFR } & \text { dihydroflavonol 4-reductase } \\ \text { ANS } & \text { anthocyanidin synthase } \\ \text { UFGT } & \text { UDP-glycose: flavonoid 3-O-glycosyltransferase } \\ \text { UVR8 } & \text { UV RESISTANCE LOCUS 8 } \\ \text { COP1 } & \text { E3 ubiquitin ligase CONSTITUTIVELY PHOTOMORPHOGENIC1 } \\ \text { HY5 } & \text { bZIP transcription factor ELONGATED HYPOGOTYL5 } \\ \text { MBW } & \text { MYB-bHLH-WD40 } \\ \text { MAPKs } & \text { mitogen-activated protein kinases } \\ \text { DPI } & \text { diphenyleneiodonium chloride } \\ \text { PVPP } & \text { polyvinylpolypyrrolidone } \\ \text { BSA } & \text { bovine serum albumin } \\ \text { DTT } & \text { dithiothreitol } \\ \text { HPLC } & \text { high-performance liquid chromatography } \\ \text { qRT-PCR } & \text { quantitative real-time polymerase chain reaction } \\ \text { UV-B } & \text { ultraviolet-B } \\ \text { ROS } & \text { reactive oxygen species } \\ \text { VS } & \text { visible sight }\end{array}$

\section{References}

1. Jimenez-Garcia, S.N.; Guevara-Gonzalez, R.G.; Miranda-Lopez, R.; Feregrino-Perez, A.A.; Torres-Pacheco, I.; Vazquez-Cruz, M.A. Functional properties and quality characteristics of bioactive compounds in berries: Biochemistry, biotechnology, and genomics. Food Res. Int. 2013, 54, 1195-1207. [CrossRef]

2. Sivankalyani, V.; Feygenberg, O.; Diskin, S.; Wright, B.; Alkan, N. Increased anthocyanin and flavonoids in mango fruit peel are associated with cold and pathogen resistance. Postharvest Biol. Technol. 2015, 111, 132-139. [CrossRef]

3. Jezek, M.; Zörb, C.; Merkt, N.; Geilfus, C.M. Anthocyanin management in fruits by fertilization. J. Agric. Food Chem. 2018, 66, 753-764. [CrossRef] [PubMed]

4. Shang, Y.; Venail, J.; Mackay, S.; Bailey, P.C.; Schwinn, K.E.; Jameson, P.E.; Martin, C.R.; Davies, K.M. The molecular basis for venation patterning of pigmentation and its effect on pollinator attraction in flowers of Antirrhinum. New Phytol. 2011, 189, 602-615. [CrossRef] [PubMed]

5. Harborne, J.B.; Williams, C.A. Advances in flavonoid research since 1992. Phytochemistry 2000, 55, 481-504. [CrossRef]

6. Cassidy, A.; Mukamal, K.J.; Liu, L.; Franz, M.; Eliassen, A.H.; Rimm, E.B. High anthocyanin intake is associated with a reduced risk of myocardial infarction in young and middle-aged women. Circulation 2013, 127, 188-196. [CrossRef]

7. Fairlie-Jones, L.; Davison, K.; Fromentin, E.; Hill, A.M. The effect of anthocyanin-rich foods or extracts on vascular function in adults: a systematic review and meta-analysis of randomised controlled trials. Nutrients 2017, 9, 908. [CrossRef]

8. Xiao, J.B.; Hogger, P. Dietary polyphenols and type 2 diabetes: current insights and future perspectives. Curr. Med. Chem. 2015, 22, 23-38. [CrossRef]

9. Tsuda, T. Dietary anthocyanin-rich plants: biochemical basis and recent progress in health benefits studies. Mol. Nutr. Food Res. 2012, 56, 159-170. [CrossRef]

10. Pojer, E.; Mattivi, F.; Johnson, D.; Stockley, C.S. The case for anthocyanin consumption to promote human health: a review. Compr. Rev. Food Sci. Food Saf. 2013, 12, 483-508. [CrossRef]

11. Kamiloglu, S.; Capanoglu, E.; Grootaert, C.; Van Camp, J. Anthocyanin absorption and metabolism by human intestinal Caco-2 cells-A review. Int. J. Mol. Sci. 2015, 16, 21555-21574. [CrossRef] [PubMed] 
12. Zhang, J.; Chen, C.; Zhang, D.; Li, H.; Li, P.; Ma, F. Reactive oxygen species produced via plasma membrane NADPH oxidase regulate anthocyanin synthesis in apple peel. Planta 2014, 240, 1023-1035. [CrossRef] [PubMed]

13. Guan, L.; Dai, Z.; Wu, B.H.; Wu, J.; Merlin, I.; Hilbert, G.; Renaud, C.; Gomès, E.; Edwards, E.; Li, S.H.; et al. Anthocyanin biosynthesis is differentially regulated by light in the skin and flesh of white-fleshed and teinturier grape berries. Planta 2016, 243, 23-41. [CrossRef] [PubMed]

14. Hu, D.G.; Sun, C.H.; Zhang, Q.Y.; An, J.P.; You, C.X.; Hao, Y.J. Glucose sensor MdHXK1 phosphorylates and stabilizes MdbHLH3 to promote anthocyanin biosynthesis in apple. PLoS Genet. 2016, 12, e1006273. [CrossRef] [PubMed]

15. Henry-Kirk, R.A.; Plunkett, B.; Hall, M.; McGhie, T.; Allan, A.C.; Wargent, J.J.; Espley, R.V. Solar UV light regulates flavonoid metabolism in apple (Malus $x$ domestica). Plant Cell Environ. 2018, 41, 675-688. [CrossRef] [PubMed]

16. Lin, Y.; Wang, Y.H.; Li, B.; Li, D.N.; Li, L.; Liu, X.; Han, J.; Meng, X. Comparative transcriptome analysis of genes involved in anthocyanin synthesis in blueberry. Plant Physiol. Biochem. 2018, 127, 561-572. [CrossRef]

17. Wang, Y.; Wang, N.; Xu, H.; Jiang, S.; Fang, H.; Zhang, T.; Su, M.; Zhang, Z.; Chen, X. Nitrogen affects anthocyanin biosynthesis by regulating MdLOB52 downstream of MdARF19 in callus cultures of red-fleshed apple (Malus sieversii f. niedzwetzkyana). J. Plant Growth Regul. 2018, 37, 719-729. [CrossRef]

18. Jaakola, L. New insights into the regulation of anthocyanin biosynthesis in fruits. Trends Plant Sci. 2013, 18, 477-483. [CrossRef]

19. Yin, R.; Skvortsova, M.Y.; Loubéry, S.; Ulm, R. COP1 is required for UV-B-induced nuclear accumulation of the UVR8 photoreceptor. Proc. Natl. Acad. Sci. USA 2016, 113, E4415-E4422. [CrossRef]

20. Li, Y.Y.; Mao, K.; Zhao, C.; Zhao, X.Y.; Zhang, H.L.; Shu, H.R.; Hao, Y.J. MdCOP1 ubiquitin E3 ligases interact with $M d M Y B 1$ to regulate light-induced anthocyanin biosynthesis and red fruit coloration in apple. Plant Physiol. 2012, 160, 1011-1022. [CrossRef]

21. Peng, T.; Saito, T.; Honda, C.; Ban, Y.; Kondo, S.; Liu, J.H.; Hatsuyama, Y.; Moriguchi, T. Screening of UV-B-induced genes from apple peels by SSH: possible involvement of MdCOP1-mediated signaling cascade genes in anthocyanin accumulation. Physiol. Plant 2013, 148, 432-444. [CrossRef] [PubMed]

22. An, J.P.; Qu, F.J.; Yao, J.F.; Wang, X.N.; You, C.X.; Wang, X.F.; Hao, Y.J. The bZIP transcription factor MdHY5 regulates anthocyanin accumulation and nitrate assimilation in apple. Hortic. Res. 2017, 4, 17023. [CrossRef] [PubMed]

23. Allan, A.C.; Hellens, R.P.; Laing, W.A. MYB transcription factors that colour our fruit. Trends Plant Sci. 2008, 13, 99-102. [CrossRef] [PubMed]

24. Li, S. Transcriptional control of flavonoid biosynthesis: fine-tuning of the MYB-bHLH-WD40 (MBW) complex. Plant Signal. Behav. 2014, 9, e27522. [CrossRef] [PubMed]

25. Xie, X.B.; Li, S.; Zhang, R.F.; Zhao, J.; Chen, Y.C.; Zhao, Q.; Yao, Y.X.; You, C.X.; Zhang, X.S.; Hao, Y.J. The bHLH transcription factor MdbHLH3 promotes anthocyanin accumulation and fruit colouration in response to low temperature in apples. Plant Cell Environ. 2012, 35, 1884-1897. [CrossRef]

26. Ju, Z.G.; Yuan, Y.B.; Liou, C.L.; Xin, S.H. Relationships among phenylalanine ammonia-Iyase activity, simple phenol concentrations and anthocyanin accumulation in apple. Sci. Hortic. 1995, 61, 215-226. [CrossRef]

27. Ju, Z.; Liu, C.; Yuan, Y. Activities of chalcone synthase and UDPGal: flavonoid-3-o-glycosyltransferase in relation to anthocyanin synthesis in apple. Sci. Hortic. 1995, 63, 175-185. [CrossRef]

28. Zhang, X.H.; Zheng, X.T.; Sun, B.Y.; Peng, C.L.; Chow, W.S. Over-expression of the CHS gene enhances resistance of Arabidopsis, leaves to high light. Environ. Exp. Bot. 2017, 154, 33-43. [CrossRef]

29. Li, P.; Ma, F.; Cheng, L. Primary and secondary metabolism in the sun-exposed peel and the shaded peel of apple fruit. Physiol. Plant 2013, 148, 9-24. [CrossRef]

30. Johnson, E.T.; Ryu, S.; Yi, H.; Shin, B.; Cheong, H.; Choi, G. Alteration of a single amino acid changes the substrate specificity of dihydroflavonol 4-reductase. Plant J. 2001, 25, 325-333. [CrossRef]

31. Shin, D.H.; Choi, M.G.; Kang, C.S.; Park, C.S.; Choi, S.B.; Park, Y.I. Overexpressing the wheat dihydroflavonol 4-reductase gene TaDFR increases anthocyanin accumulation in an Arabidopsis dfr mutant. Genes Genom. 2015, 38, 333-340. [CrossRef]

32. Gu, Z.; Chen, H.; Yang, R.; Ran, M. Identification of DFR as a promoter of anthocyanin accumulation in poinsettia (Euphorbia pulcherrima, willd. ex Klotzsch) bracts under short-day conditions. Sci. Hortic. 2018, 236, 158-165. [CrossRef] 
33. Ubi, B.E.; Honda, C.; Bessho, H.; Kondo, S.; Wada, M.; Kobayashi, S.; Moriguchi, T. Expression analysis of anthocyanin biosynthetic genes in apple skin: effect of UV-B and temperature. Plant Sci. 2006, 170, 571-578. [CrossRef]

34. Ban, Y.; Honda, C.; Hatsuyama, Y.; Igarashi, M.; Bessho, H.; Moriguchi, T. Isolation and functional analysis of a MYB transcription factor gene that is a key regulator for the development of red coloration in apple skin. Plant Cell Physiol. 2007, 48, 958-970. [CrossRef]

35. Plunkett, B.J.; Espley, R.V.; Dare, A.P.; Warren, B.A.W.; Grierson, E.R.P.; Cordiner, S.; Turner, J.L.; Allan, A.C.; Albert, N.W.; Davies, K.M.; et al. MYBA from blueberry (Vaccinium Section Cyanococcus) is a subgroup 6 type R2R3MYB transcription factor that activates anthocyanin production. Front. Plant Sci. 2018, 9, 1300. [CrossRef]

36. Hideg, É.; Jansen, M.A.; Strid, Å. UV-B exposure, ROS, and stress: inseparable companions or loosely linked associates? Trends Plant Sci. 2013, 18, 107-115. [CrossRef]

37. Wargent, J.J.; Jordan, B.R. From ozone depletion to agriculture: understanding the role of UV radiation in sustainable crop production. New Phytol. 2013, 197, 1058-1076. [CrossRef]

38. Chen, C.S.; Zhang, D.; Wang, Y.Q.; Li, P.M.; Ma, F.W. Effects of fruit bagging on the contents of phenolic compounds in the peel and flesh of 'Golden Delicious', 'Red Delicious', and 'Royal Gala' apples. Sci. Hortic. 2012, 142, 68-73. [CrossRef]

39. Zhang, J.; Niu, J.; Duan, Y.; Zhang, M.; Liu, J.; Li, P.; Ma, F. Photoprotection mechanism in the 'Fuji' apple peel at different levels of photooxidative sunburn. Physiol. Plant 2015, 154, 54-65. [CrossRef]

40. Lister, C.E.; Lancaster, J.E.; Sutton, K.H.; Walker, J.R.L. Aglycone and glycoside specificity of apple skin flavonoid glycosyltransferase. J. Sci. Food Agric. 1997, 75, 378-382. [CrossRef]

41. Bi, X.; Zhang, J.; Chen, C.; Zhang, D.; Li, P.; Ma, F. Anthocyanin contributes more to hydrogen peroxide scavenging than other phenolics in apple peel. Food Chem. 2014, 152, 205-209. [CrossRef] [PubMed]

42. Li, P.; Cheng, L. The elevated anthocyanin level in the shaded peel of 'Anjou' pear enhances its tolerance to high temperature under high light. Plant Sci. 2009, 177, 418-426. [CrossRef]

43. Malnoy, M.; Reynoird, J.P.; Mourgues, F.; Chevreau, E.; Simoneau, P. A method for isolating total RNA from pear leaves. Plant Mol. Biol. Rep. 2001, 19, 69. [CrossRef]

(C) 2019 by the authors. Licensee MDPI, Basel, Switzerland. This article is an open access article distributed under the terms and conditions of the Creative Commons Attribution (CC BY) license (http://creativecommons.org/licenses/by/4.0/). 